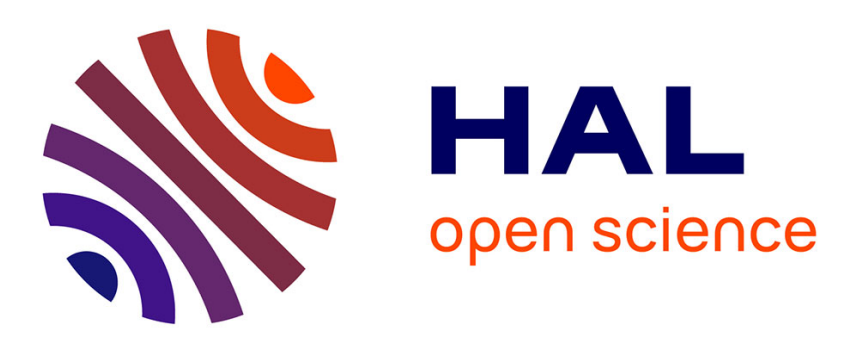

\title{
On-line drilling process monitoring by Marginalized Particle Filter
}

\author{
Amadou Ba, Nazih Mechbal, Michel Vergé, Slim Hbaieb
}

\section{To cite this version:}

Amadou Ba, Nazih Mechbal, Michel Vergé, Slim Hbaieb. On-line drilling process monitoring by Marginalized Particle Filter. 2009 IEEE Aerospace Conference, 2009, Montana, United States. 10.1109/AERO.2009.4839475 . hal-00362543

\section{HAL Id: hal-00362543 https://hal.science/hal-00362543}

Submitted on 20 Apr 2018

HAL is a multi-disciplinary open access archive for the deposit and dissemination of scientific research documents, whether they are published or not. The documents may come from teaching and research institutions in France or abroad, or from public or private research centers.
L'archive ouverte pluridisciplinaire HAL, est destinée au dépôt et à la diffusion de documents scientifiques de niveau recherche, publiés ou non, émanant des établissements d'enseignement et de recherche français ou étrangers, des laboratoires publics ou privés. 


\title{
On-Line Drilling Process Monitoring by Marginalized Particle Filter
}

\author{
${ }^{1-2} \mathrm{~A}$. BA, ${ }^{1} \mathrm{~N}$. Mechbal, ${ }^{1} \mathrm{M}$. Vergé, ${ }^{2} \mathrm{~S}$. Hbaieb, \\ ${ }^{1}$ Laboratoire de Mécanique des Systèmes et des Procédés (UMR-CNRS), \\ Ecole Nationale Supérieure d'Arts et Métiers, 151, Boulevard de l'hôpital, Paris, France \\ ${ }^{2}$ Schlumberger Riboud Product Centre, 1, rue Henri Becquerel, 92142 Clamart, Cedex, France \\ $331-45-37-70-87$ \\ aba2@slb.com
}

Abstract - Real-time monitoring of a drilling process is an essential task in improving their performances. ${ }^{12}$ Faults that might occur have to be detected as soon as possible in order to preserve drilling efficiency. In this paper, drilling process monitoring by identifying time varying parameters through Marginalized Particle Filter (MPF) is treated. The idea consists in enhancing the tracking ability of parameters change by integrating into the process model a part that represents the faulty process and another when the process is safe. The efficiency of the developed approach is highlighted through simulated and experimental data obtained from tests campaign.

\section{TABLE OF CONTENTS}

1. INTRODUCTION

2. BIT ROCK INTERACTION MODEL

3. PARTICLES FILTER .............................................................2

4. LINEAR REGRESION AND FD BY MPF .................................3

5. LINEAR REGRESION AND FD BY MPF AND SIMULATED

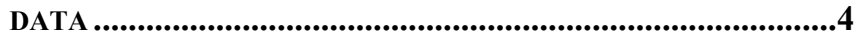

6. LINEAR REGRESION AND FD BY MPF AND EXPERIMENTAL

DATA ........................................................................................





REFERENCES ..................................................................6

\section{INTRODUCTION}

Drilling process performances are adversely affected when a fault occurs. For this reason, a system for the faults detection (FD) plays a vital role. It may enable to be able to safeguard some of the process performances by taking efficient corrective action, in case of early diagnosis. Drilling processes are very widespread in several fields and their efficiencies are more and more required, owing to the hostile environments that they are confronted and to the expense of maintenance procedure. As an example, oilfield industry necessitates to have an efficient drilling process (Fig.1(a)) in order to drill in a more challenging context, such as high temperature, high pressure, heterogeneous rock, among others problematic cases. Aerospace domain (Fig.1(b)) uses a robot that integrates a drill bit for planetary exploration, potentially to look for evidence of possible life
[1]. All these cases require an efficient diagnosis system to detect in real time faults that might occur. This paper deals with a monitoring strategy to detect poor performances of the drill bit. When this scenario happens, the drilling operation cannot be achieved as expected. In this paper, the FD strategy investigated concerns a drilling process used in oilfield industry. Nevertheless, the developed approach can be experimented for other drilling processes, especially those used in aerospace technology. Indeed, there is no doubt that the differences in processes lead to the discrepancies in their dynamics. But, the principle of the bit rock interaction in both cases shows great similarities. Any of these processes requires, at the bit, two forces to grind the rock: the Weight On Bit (WOB) and the Torque On Bit $(T O B)$. In this study, the fault to detect is characterized by the change in the drill bit properties, consequently in forces values for a constant Rate Of Penetration (ROP). It is interesting to make clear that the change in rock properties, for example, moving from slight to hard rock, provides similar behavior in measurement compared with those viewed in the case where the drill bit is faulty. Here, the question is how to dissociate these two phenomena? To provide an answer to this question it is developed in [2] a model of bit rock interaction. This model gives the possibility to express forces between them without involving the rock properties. When a fault occurs on the drill bit, the coefficients which characterize these relationships will change. The idea is to monitor these coefficients and to detect their possible modification. To achieve this task, we propose to investigate MPF in order to detect faults might occurs in the bit. The FD approach proposed is based on the principle which consists in defining two functioning modes, one corresponding to the case where the process is safe and another when it is faulty. Each of the defined functioning modes is modeled by a state space representation system. Through this approach, the classic identification methods provide poor performances. For this reason, we investigate the MPF. Its specificity comes from the possibility to be able to combine the features of the Kalman Filter (KF) [3] and those provided by the Particle Filter (PF) [4]. The role of the KF is to treat each of the defined state space representation whereas the $\mathrm{PF}$ is dedicated to select the one to be treated. The FD strategy is achieved through two linear regressions obtained by using MPF approach and the detection is made when the process model move from the model which corresponds to the process when it is safe to the faulty case. In this paper, 
our study is restricted to two possible classes in which the process can evolve, but the
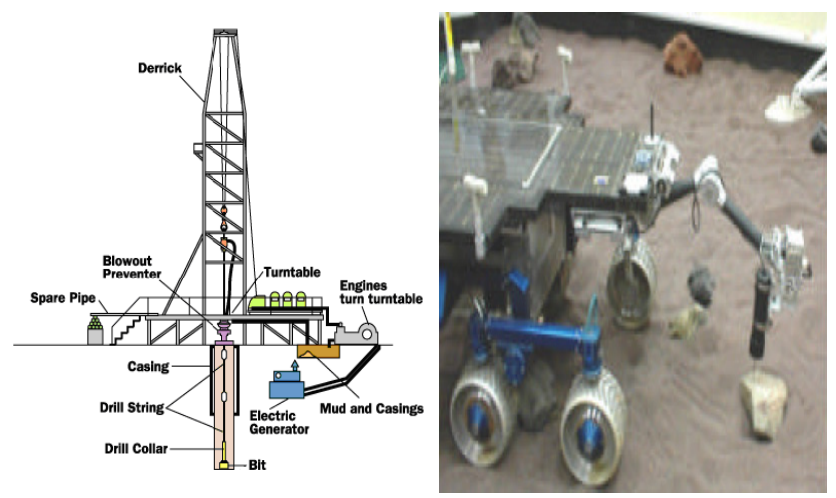

Fig.1(a) Oilfield drilling process Fig.1 (b) Robot with drilling process

study can be extended to other classes. Before the use of MPF for linear regression and FD, we briefly present the model of bit-rock interaction as developed in [2].

\section{BIT ROCK INTERACTION MODEL}

The model of bit rock interaction has been subject of intensive researches activities and interesting results are reported in [2]. These models account for the cutting action of a single cutter. Cutting action of each cutter is represented by two independent processes, the cutting process and the friction process. The forces $T O B$ and $W O B$ are defined by:

$$
T O B=T O B_{c}+T O B_{f}, \quad W O B=W O B_{c}+W O B_{f}
$$

where:

$$
\begin{aligned}
& T O B_{\mathrm{c}}=\frac{\varepsilon \cdot a^{2} \cdot D O C a}{2}, W O B_{\mathrm{c}}=\xi \cdot \varepsilon \cdot a \cdot D O C \\
& T O B_{f}=\frac{\mu \cdot a \cdot \gamma \cdot W O B_{\mathrm{f}}}{2}, D O C=\frac{2 \cdot \pi \cdot R O P}{\Omega}
\end{aligned}
$$

The subscripts $c$ and $f$ denote cutting and friction respectively. The cutting components $T O B_{\mathrm{c}}$ and $W O B_{\mathrm{c}}$ correspond to the forces transmitted by the cutting face of each cutter and the friction components $T O B_{f}$ and $W O B_{f}$ correspond to the other contacts obtained from bit-rock interaction. The response of the bit is obtained by combining the cutting and frictional process [2]:

$$
\frac{2 \cdot T O B}{a^{2} \cdot D O C}=(1-\beta) \cdot \varepsilon+\mu \cdot \gamma \cdot \frac{W O B}{a \cdot D O C}
$$

with:

$$
\beta=\mu \cdot \gamma \cdot \zeta
$$

The specific energy which corresponds to the necessary energy to grind a given volume of rock is defined in [2] by:

$$
E=E_{0}+\mu \cdot \gamma \cdot S
$$

where:

$$
E_{0}=(1-\beta) \cdot \varepsilon
$$

Through (1) and (2) the following relations have been obtained:

$$
S=\frac{W O B}{a \cdot D O C}, E=\frac{T O B}{a^{2} \cdot D O C}
$$

The relationship (2) shows that the slope of $E$ as a function of $S$ is only dependent on the bit shape factor and the friction coefficient. Hence, the rock properties are not involved.

When a change occurs on the bit, for example the bit wear or break, these two parameters will be affected. Consequently, the fault detection approach developed in this study is based on the detection of a change in $\mu$ and / or $\gamma$. That leads to the change in the slope of $E$ as a function of $S$

\begin{tabular}{|c|c|c|}
\hline Symbol & Quantity & Unity \\
\hline$a$ & Bit radius & $\mathrm{m}$ \\
\hline$D O C$ & Depth of cut & $\mathrm{m}$ \\
\hline$E$ & Specific energy & Mpa \\
\hline$S$ & Drilling strength & Mpa \\
\hline$T O B$ & Torque on bit & $\mathrm{Nm}$ \\
\hline$T O B_{c}$ & Torque on bit, cut & $\mathrm{Nm}$ \\
\hline$T O B_{\mathrm{f}}$ & Torque on bit, friction & $\mathrm{Nm}$ \\
\hline$W O B$ & Weight on bit & $\mathrm{N}$ \\
\hline$W O B_{c}$ & Weight on bit, cut & $\mathrm{N}$ \\
\hline$W O B_{f}$ & Weight on bit, friction & $\mathrm{N}$ \\
\hline$\varepsilon$ & Intrinsic specific energy & Mpa \\
\hline$R O P$ & Rate of penetration (ROP) & $\mathrm{m} / \mathrm{h}$ \\
\hline$\Omega$ & Bit angular velocity & $\mathrm{rad} / \mathrm{s}$ \\
\hline$\mu$ & Friction coefficient & \\
\hline$\xi$ & Coefficient characterizing cutting force & \\
\hline$\gamma$ & Bit shape factor & \\
\hline
\end{tabular}
as indicated in (2). Here, the model (2) is exploited in order to monitor the drill bit. For this reason, MPF algorithm is used in order to identify the parameters corresponding to the two slopes, before and after the change, as well as the time from which the change occurs.

TABLE I

VARIABLES USED

\section{PARTICLES FILTER}

In this paper it is not intended to provide a detail regarding the theory of PF. The interested reader is refereed to [5]. To make easy the understanding of the MPF approach we present the idea of PF. The PF is a statistic tool which approximates a probability density function trough simulation. To achieve this aim, the PF algorithm requires a large number of samples called particles in order to represent accurately the real state of the process. At each time, the samples generation is needed. When a measurement becomes available weights are assigned to the 
probable state. It can be considered as the degree of confidence that we need to have on the particles in their representation of the true state of the process. The weight values depend on how they fit the measurements as well as the sequence of the past measurements. Only, the most likely particles to represent the true states are maintained onto the next step and the other are discarded through the resampling algorithm. As an extension of the PF, the MPF algorithm [6-8] is applicable to the case where the dependencies within the states variables can be analytically used. To illustrate that, let us consider the case where two process models can be defined, one representing the process when it is safe whereas the other when it is faulty, and each of the model having a linear state space representation. It is obviously clear that the two models cannot be exploited at the same time; as a result one of them is selected when it is considered as the most probable scenario by the PF. In this context, the standard KF is used for the two linear state space models, and the PF to select the one to be involved. To formalize this situation we consider the following state space representation:

$$
\begin{aligned}
& x_{k+I}^{2}=A_{k} \cdot x_{k}^{2}+G_{k} \cdot \omega_{k} \\
& y_{k}=C_{k} \cdot x_{k}^{2}+H_{k} \cdot v_{k}
\end{aligned}
$$

Where $x_{k}^{2}$ is considered to model the evolution of the process. As suggested in [9], it is important to make clear that the model can possess one or more of the matrices $A_{k}, G_{k}, C_{k}$ and $H_{k}$ evolving possibly under nonlinear, non-Gaussian conditions. The variables $v_{k}$ and $\omega_{k}$ are considered zero mean and Gaussian. As matrices in this system can change according to the state in which the process progress, solving the problem requires to achieve another set of variables, describing the evolutions of these parameters. This context brings us to define by $x_{k}^{1}$ as this set of parameters. Thereby, the aims becomes the use of the MPF for the overall state: $x_{k}=\left\{\begin{array}{ll}x_{k}^{1} & x_{k}^{2}\end{array}\right\}$. In order to achieve this requirement, it is essential to define the transition probability $P\left(x_{k}^{1} / x_{k-1}^{1}\right)$ between these two states and to have a priori knowledge concerning the variable $x_{k}^{1}$. At last, to generate samples from $P\left(x_{k}^{1} / x_{k-1}^{1}\right)$. It can be noticed that the $\mathrm{KF}$ only do not enables to solve this kind of problem. Therefore, there is necessity to have, at each time an algorithm estimating $x_{k}^{1}$ to serve the KF to run $x_{k}^{2}$. To accomplish this objective, a set of $\mathrm{N}$ particles is generated at every time step, maintained and updated. As an example at the time $k$, the $\mathrm{i}^{\text {th }}$ particle is defined as $\left\{x_{k}^{1,(i)} ; x_{k}^{2,(i)} ; K_{k}^{(i)}\right\}$ where $K_{k}^{(i)}$ corresponds to the covariance of $x_{k}^{2,(i)}$ given the set $X_{k}^{1,(i)}=\left\{x_{r}^{1,(i)}\right\}_{r=0}^{k}$. For each iteration, $x_{k}^{1,(i)}$ corresponds to a unique $A_{k}^{(i)}, G_{k}^{(i)}, C_{k}^{(i)}$ and $H_{k}^{(i)}$.

\section{LINEAR REGRESION AND FD BY MPF}

The objective of this study is to perform a FD task by using MPF. The FD approach developed here is based on parameter estimation technique particularly on linear regression method. The aim is to detect a change, which corresponds to the fault apparition, in the slope progress. To perform the MPF approach we define two slopes where one represents the process in the case where it is safe and another when it is faulty. We model these two slopes by two state space representations, the algorithm switch from one model to another through a variable which evolves under PF principles. For the reason of clarity, we redefine the model (3) under classic state space linear regression form. That is, we replace $x_{k}^{2}$ by $\theta, x_{k}^{1}$ by $z_{k}, A_{k}$ by identity matrix and $C_{k}$ by $\varphi_{k}^{T}$. Thus we get the following state space representation:

$$
\begin{aligned}
& z_{k} \sim p\left(z_{k} / z_{k-1}\right) \\
& \theta_{k+1}=\theta_{k}+G_{k}\left(z_{k}^{(i)}\right) \cdot \omega_{k} \\
& y_{k}=\varphi_{k}^{T}\left(z_{k}^{(i)}\right) \cdot \theta_{k}+H_{k}\left(z_{k}^{(i)}\right) \cdot v_{k}
\end{aligned}
$$

Where $z_{k} \in\left\{1, \cdots, n_{z}\right\}$ denotes the unknown state: Here the safe state is considered when $z_{k}=1$ and the faulty state when $z_{k}=2 . \theta \in \mathfrak{R}^{n} \theta$ is the parameter vector, $\mathrm{y} \in \mathfrak{R}^{\mathrm{n}_{\mathrm{y}}}$ is the measurement vector. $\omega_{k}$ and $v_{k}$ are respectively the process noise and the measurement noise, considered to be known, independent and identically distributed (i.i.d). $\varphi_{k}^{T}$ is the regressor vector, (i) is the particle associated to the model, $G_{k}$ and $H_{k}$ are the matrices associated to the noises. As said above, two states of the process are defined, thus $z_{k}^{(i)} \in\{1,2\}$ According to the $\mathrm{z}_{\mathrm{k}}^{(\mathrm{i})}$ value one of the models is involved. Notice that the purpose of the MPF approach is to estimate the following probability density function:

$$
p\left(\theta_{k}, z_{k} / Y_{k}\right)=p\left(\theta_{k} / z_{k}, Y_{k}\right) \cdot p\left(z_{k} / Y_{k}\right)
$$

In the equation (5), $Y_{k}$ denotes the sequence of measurements up to time k, $Y_{k}=\left\{y_{0}, \cdots, y_{k}\right\}$. From this equation, it is clear that the PF will be used for estimating the probability density function $p\left(z_{k} / Y_{k}\right)$, and the KF is employed to estimate $p\left(\theta_{k} / z_{k}, Y_{k}\right)$. The model of equation (4) enables to define the algorithm- 1 . In this algorithm the main step are given. At the starting, $\mathrm{N}$ particles are drawn according to $z_{0 /-1}^{(i)} \sim p\left(z_{0}\right)$. And the KF associated with each parameter sample are initialized as:

$\left\{\theta_{0 /-1}^{(i)}, P_{0 /-1}^{(i)}\right\}=\left\{\theta_{0}, P_{0}\right\}_{i=1}^{N}$

Then weights are evaluated and normalized according to: $\left.q_{k}^{(i)}=p\left(y_{k} / Y_{k}^{(i)}, z_{k / k-1}^{(i)}\right)\right) \sim N\left(y_{k / k-1}^{(i)}, S_{k}^{(i)}\right)$ where $S_{k}^{(i)}$ is the innovation term associated to each particles. After that, the particles having the highest weight are multiplied and those 
having the lowest weight are discarded through the resampling algorithm defined in the algorithm-2.

Then the particles are updated by using the KF. At the end, the parameter vector is estimated. In the algorithm 1 $R_{k}$ denotes the variance of the noise measurement $v_{k}, Q_{k}$ is the covariance of process noise $\omega_{\mathrm{k}}$.

Algorithm: MPF for FDI

1) for $i=1, \ldots, N$, Propagate samples

$$
\begin{aligned}
z_{0 /-1}^{(i)} & \sim p\left(z_{0} / z_{0 /-1}^{(i)}, Y_{0}\right) \\
& =p\left(Y_{0} / z_{0}\right) \cdot p\left(z_{0} / z_{0 /-1}^{(i)}\right)
\end{aligned}
$$

and set $\left\{\theta_{0 /-1}^{(i)}, P_{0 /-1}^{(i)}\right\}=\left\{\theta_{0}, P_{0}\right\}_{i=1}^{N}$

end for

2) for $i=1, \ldots, N$,

a) evaluate the weight

$\left.q_{k}^{(i)}=p\left(y_{k} / Y_{k}^{(i)}, z_{k / k-1}^{(i)}\right)\right) \sim \boldsymbol{\aleph}\left(y_{k / k-1}^{(i)}, S_{k}^{(i)}\right)$

where: $y_{k / k-1}=\varphi_{k}^{T}\left(z_{k / k-1}^{(i)}\right) \cdot \theta_{k / k-1}^{(i)}$

$S_{k}^{(i)}=\varphi_{k}^{T}\left(z_{k / k-1}^{(i)}\right) \cdot P_{k / k-1}^{(i)} \cdot \varphi_{k}\left(z_{k / k-1}^{(i)}\right)+R_{k}$

b) Normalize the weight

$\bar{q}_{k}^{(i)}=\frac{q_{k}^{(i)}}{\sum_{j=1}^{N} q_{k}^{(j)}}$

end for

3) Resample with replacement, (Algorithm2)

$P\left(z_{k / k}^{(i)}=z_{k / k-1}^{(j)}\right)=\bar{q}_{k}^{(j)}$

4) Kalman filter measurements update

equation (6)

5) Compute the parameters vector equation (7)

The equation (6) is given by:

$$
\begin{aligned}
& S_{k}^{(i)}=R_{k}+\varphi_{k}^{T}\left(z_{k / k}^{(i)}\right) \cdot P_{k / k-1}^{(i)} \cdot \varphi_{k}\left(z_{k / k}^{(i)}\right) \\
& K_{k}^{(i)}=P_{k / k-1}^{(i)} \cdot \varphi_{k}^{T}\left(z_{k / k}^{(i)}\right) \cdot\left(S_{k}^{(i)}\right)^{-1} \\
& \theta_{k / k}^{(i)}=\theta_{k / k-1}^{(i)}+K_{k}^{(i)}\left(y_{k}-\varphi_{k}^{T}\left(z_{k / k}^{(i)}\right) \cdot \theta_{k / k-1}^{(i)}\right) \\
& P_{k / k}^{(i)}=P_{k / k-1}^{(i)}-K_{k}^{(i)} \cdot S_{k}^{(i)} \cdot\left(K_{k}^{(i)}\right)^{T}
\end{aligned}
$$

In the equation (6) $K_{k}^{(i)}$ is the Kalman gain which minimizes the posterior error covariance, $S_{k}^{(i)}$ is the innovation matrix, $R_{k}$ denotes the variance of the noise measurement $v_{k}$.

Algorithm 2: Resampling algorithm

- Generate an uniformly distributed random point $\mathrm{u}_{1} \in\left[0, \mathrm{~N}^{-1}\right]$ and let $\mathrm{i}=1, \mathrm{q}_{\mathrm{k}}^{0}=0$

- $\quad$ For $\mathrm{j}=1: \mathrm{N}$

1. Let $\mathrm{u}_{\mathrm{j}}=\mathrm{u}_{1}+\mathrm{N}^{-1} \cdot(\mathrm{j}-1)$

2. If $\sum_{\mathrm{l}=0}^{\mathrm{i}-1} \mathrm{q}_{\mathrm{k}}^{1}<\mathrm{u}_{\mathrm{j}} \leq \sum_{\mathrm{l}=0}^{\mathrm{i}} \mathrm{q}_{\mathrm{k}}^{1}$ set $\mathrm{x}_{\mathrm{k}}^{\mathrm{j}}=\mathrm{x}_{\mathrm{k} / \mathrm{k}-1}^{\mathrm{i}}$

\section{Otherwise go to step (3) - $\underline{\text { Algorithm1 }}$}

- $\quad$ End for

The resampling step is achieved according to the algorithm2 , during this stage the particles having the highest weight are multiplied and those having the lowest weight are discarded.

\section{LINEAR REGRESION AND FD BY MPF AND SIMULATED DATA}

In the past sections we described the MPF approach and the use of this technique for FD task. The proposed FD strategy uses the model selection approach, where a PF choose between two defined models, one representing the process running in normal operation and other for the faulty process. The advantage of such approach compared with the classical identification technique comes from the fact that the fault is detected by a discrete state as soon as possible. Here we are concerned to detect the change in the slope which characterizes a fault apparition by using MPF. We start by appraising this approach, in its ability to be able to detect fault, by using simulated data: Let us take two equations as defined in (7) and (8). We consider that ones corresponds to the case where there is no fault (7) and the other when the process is faulty (8). To these equations it is added the measurements noises, denoted by e:

$$
\begin{aligned}
& y_{1}=0.03 \cdot x+1+\text { rand } \\
& y_{2}=0.015 \cdot x+12+\text { rand }
\end{aligned}
$$

These equations can be rewritten as:

$$
y_{1}=\left[\begin{array}{ll}
x & 1
\end{array}\right] \cdot\left[\begin{array}{c}
0.03 \\
1
\end{array}\right]+\text { rand } y_{2}=\left[\begin{array}{ll}
x & 1
\end{array}\right] \cdot\left[\begin{array}{c}
0.015 \\
12
\end{array}\right]+\text { rand }
$$

In general term, we get for each model the following state space representation:

$$
\left\{\begin{array}{c}
\theta_{1 k+1}=\theta_{1 k}+\omega_{1 k} \\
y_{1 k}=\varphi_{1 k}^{T} \cdot \theta_{1 k}+e_{1 k}
\end{array}, \quad\left\{\begin{array}{c}
\theta_{2 k+1}=\theta_{2 k}+\omega_{2 k} \\
y_{2 k}=\varphi_{2 k}^{T} \cdot \theta_{2 k}+e_{2 k}
\end{array}\right.\right.
$$

The MPF allows to define one general model for both cases:

$$
\begin{aligned}
& \theta_{k+1}=\theta_{k}+G_{k}\left(z_{k}^{(i)}\right) \cdot \omega_{k} \\
& y_{k}=\varphi_{k}^{T}\left(z_{k}^{(i)}\right) \cdot \theta_{k}+H_{k}\left(z_{k}^{(i)}\right) \cdot v_{k}
\end{aligned}
$$

Where $z_{k}^{(i)}$ allows to move from one model to another through the transition matrix given by $P\left(x_{k}^{1} / x_{k-1}^{1}\right)$. The aim is to use the presented algorithm in order to identify the slopes defined in (7) and (8) as well as to detect the faults. For reliable FD, robustness against measurement noise and other possible disturbance is required. For this reason we choose reasonable amplitude of rand. The Fig. 3 presents the slope obtained by using the proposed approach. Notice 
that when the fault occurs the MPF model for normal operation is substituted by the MPF model considering that fault is active. In the first example, the number of particles is equal to 50 .

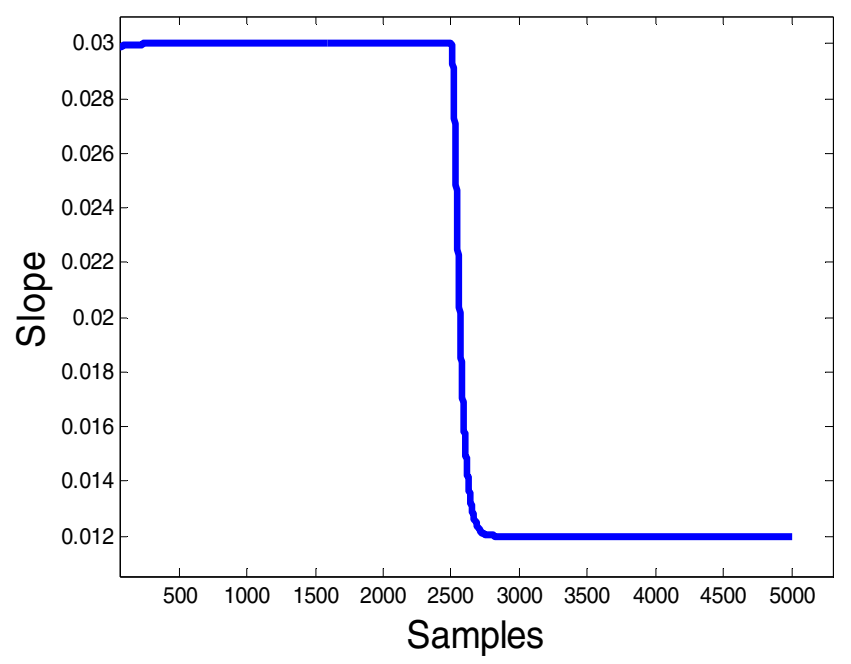

Fig.3. Estimated slope data (50 particles)

In this figure, two functioning modes can be noticed, one corresponding to the safe functioning and the other to the faulty process. Each of these two modes presents a stable regime. Between 2500 and 2700 samples the process is in transient regime. There is no fluctuation in the evolution of the slope that corresponds to the good performances of the algorithm. Here, the transient regime is achieved by the PF. In addition, the MPF shows good performances in terms of rapidity to detection, convergence to the value which characterizes the fault, among others. Its performances depend on the number of particles. When the number of particles is increased the duration of the transient stage is reduced as shown in Fig. 4.

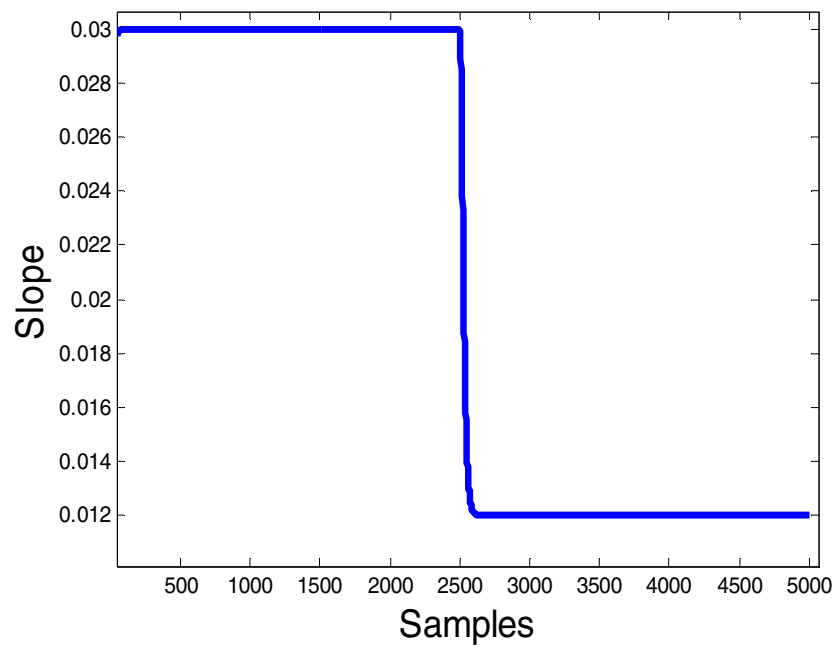

\section{LINEAR REGRESION AND FD BY MPF AND EXPERIMENTAL DATA}

To emphasize the possibility and to show the ability of the MPF algorithm to perform parameter estimation and fault detection scheme, an illustrative actual application is presented in this section. This application uses real experimental data. The aim is to detect a fault often encountered in oilfield industry. This fault concerns a head of a drilling process (drill bit), in other words, it occurs on the part of the drilling process which grinds the rock. Here, the objective is to detect the malfunction that might occur on this bit. As explained in the previous section, these malfunctions are described by a change in a parametric slope. To understand how this kind of dysfunction can interfere with process operation, plots of $E, S$ and $R O P$ over time are given in Fig. 5. Before sample number 1500, ROP was adjusted continuously to find a set of points that allows to construct a slope. It can be noticed that during this samples interval $S$ is almost constant. It is due to the fact that dysfunction has not yet occurred. In this case, the mean value of the drilling strength comes from rock hardness. In spite of rock homogeneity and a constant $R O P$ in the sample interval between [2450 5000] $S$ and $E$ increases. These increases come from $T O B$ and $W O B$ and are due to the change in the friction coefficient $\mu$ or/and the bit shape factor $\gamma$. These changes reveal a dysfunction on the bit. However, in real drilling conditions it is not a straightforward task to diagnose this kind of faults by looking at the progress in measurements. It is mainly caused by the similar behavior viewed on $\mathrm{S}$ and $\mathrm{E}$ either in case of changeover from slight rock to hard rock or the bit dysfunction. For this reason, we have developed an on-line identification method to determine the slope which characterizes $\mathrm{E}$ as a function of $\mathrm{S}$ and consequently detecting the dysfunction on the bit.
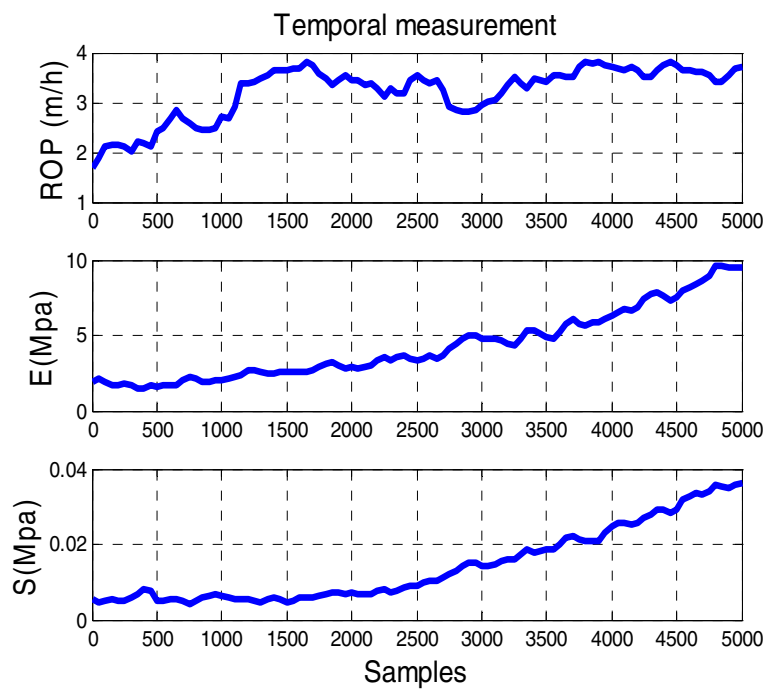

Fig. 5. Behavior of measurements in case of dysfunction on the drill bit

Fig.4. Slopes progress simulated data (100 particles) 


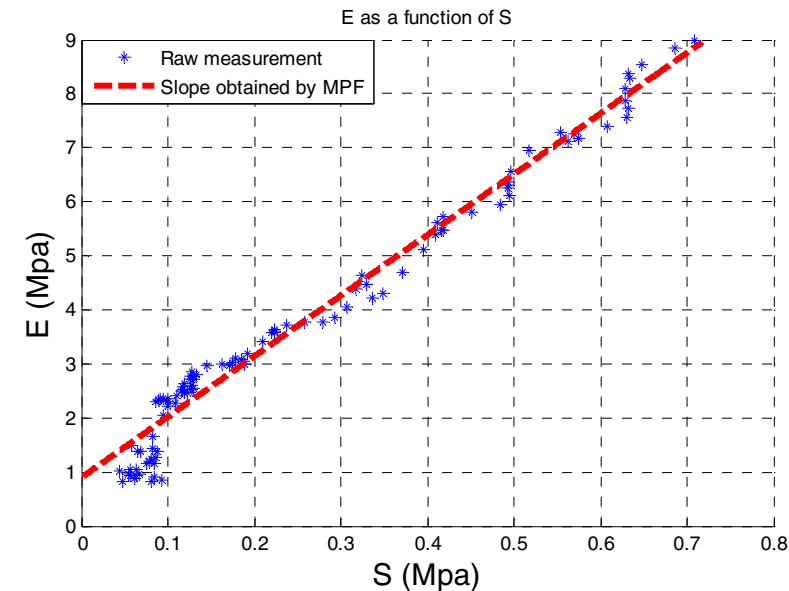

Fig.6. E as a function of S-raw data- and linear regression by MPF

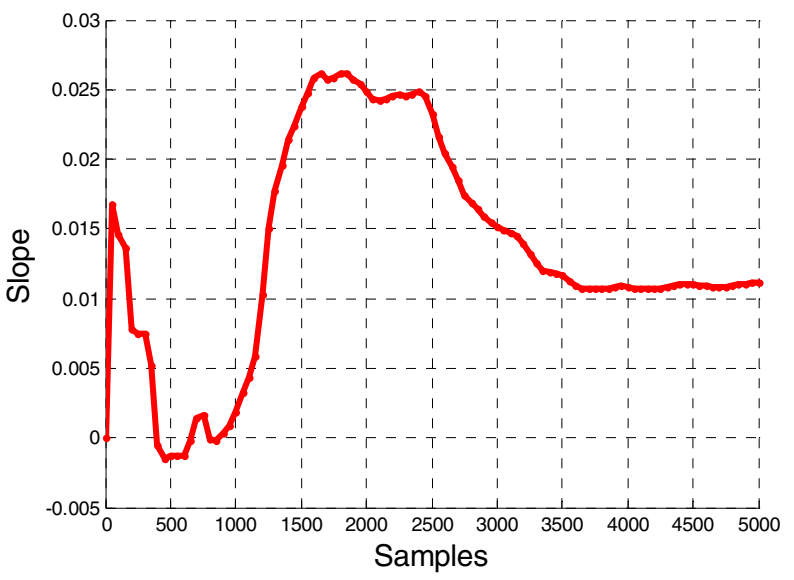

Fig.7. Slopes progress real data (50 particles)

The Fig.6 presents an example of application of the MPF algorithm by using experimental data. It can be seen from this figure the raw measurement and the slope obtained by using MPF. The Fig.7 presents the temporal evolution of the slope in the case where the number of particles is equal to 50. It can be seen from this figure two regimes of functioning the first one corresponds to the absence of faults, in this case the mean value of the slope is equal to 0.025 and the second corresponds to the case where there is fault the mean value of the slope becomes equal to 0.012 .

\section{CONCLUSIONS}

A FD approach based on the MPF is studied in this paper. The approach proposed uses two models: one represents the process evolution in presence of fault and another when the process is safe. The performances of the proposed approach are highlighted by using simulated and experimental data. This approach showed interesting results in terms of rapidity in detection, convergence to the final value, as well as robustness against noises.

The requirement to diagnose bit dysfunction when it is incipient is useful concerning appropriate corrective action to take and that brings the process to an optimal drilling condition. The next step of this study is to develop a fault tolerant control algorithm in order to automatically adjust the input of the drilling process in case of bit dysfunction detection. Also, in the future research we will consider the case where several classes of faults are defined, that will help to detect and define the fault severity.

\section{ACKNOWLEDGMENT}

The authors would like to thank the Schlumberger Riboud Product centre for supporting this work.

\section{REFERENCES}

[1] G. Paulsen, K.Zacny, P. Chu, E.Mumm, K.Davis, S.Frader-Thompson, H. Cannon, B. Glass, "Robotic Drill Systems for planetary Exploration", Proc. AIAA Space 2006, San Jose, CA, September, 2006.

[2] T. Richard and E. Detournay, E. "Influence of bit rock interaction on stick slip vibrations of PDC bits", Society of Petroleum and Engineers, Texas, October, 2002.

[3] L Ljung "System identification -Theory for the user" (Prentice Hall, Upper Saddle River, NJ, 2nd edn.), 1999.

[4] A. Doucet, N.J Gordon, and V. Krishnamurthy, "Particle filters for state estimation of jump Markov linear system", IEEE Trans. Signal Process., (49), (3), pp. 613-624, 2001.

[5] A. Doucet, N. De Freitas and N. Gordon, "Sequential Monte Carlo methods in practice". Springer, Verlag 2001.

[6] T. Schön, R. Karlsson and F. Gustafsson. "The marginalized particle filter in practice". In proceedings IEEE Aerospace Conference, Big Sky, USA, Mars, 2006.

[7] T. Schön and F. Gustafsson, "Particle filters for system identification of state-space models linear in either parameters or states". In proceedings of the $13^{\text {th }}$ IFAC symposium in system identification, pages 1287-1292, Rotterdam, The Netherlands, September, 2003

[8] F. Gustafsson and P. Hriljac, "Particle filters for system identification with application to chaos prediction'. Proceedings to $13^{\text {th }}$ IFAC symposium in system identification, Rotterdam, The Netherlands, September, 2003.

[9] P. Li, R. Goodall, and V. Kadirkamanathan. "Estimation of parameters in a linear state space model using Raoblackwellised particle filter". IEEE proceedings of Control Theory Applications, 151(6):727-738, November, 2004. 